\title{
Polymer Bound Photobase Generators and Photoacid Generators for Pitch Division Lithography
}

\author{
Younjin Cho ${ }^{\mathrm{a}}$, Xinyu Gu ${ }^{\mathrm{a}}$, Yuji Hagiwara ${ }^{\mathrm{a}}$, Takanori Kawakami ${ }^{\mathrm{a}}$, \\ Toshiyuki Ogata ${ }^{\mathrm{b}}$, Brandon Rawlings ${ }^{\mathrm{a}}$, Yongjun Li ${ }^{\mathrm{c}}$, Arun K. Sundaresan ${ }^{\mathrm{c}}$, \\ Nicholas J. Turro ${ }^{c}$, Robert Bristol ${ }^{\text {d, James M. Blackwell }}{ }^{\mathrm{d}}$, \\ C. Grant Willson ${ }^{\mathrm{a}, \mathrm{b}}$ \\ ${ }^{a}$ Department of Chemical Engineering and ${ }^{b}$ Department of Chemistry, \\ The University of Texas at Austin, Austin, TX 78712, USA; \\ ${ }^{\mathrm{c} D}$ Department of Chemistry, Columbia University, New York, NY 10027, USA; \\ ${ }^{\mathrm{d}}$ Intel Corporation, TD, Hillsboro, OR 97124, USA
}

\begin{abstract}
The semiconductor industry is pursuing several process options that provide pathways to printing images smaller than the theoretical resolution limit of $193 \mathrm{~nm}$ projection scanners. These processes include double patterning, side wall deposition and pitch division. Pitch doubling lithography (PDL), the achievement of pitch division by addition of a photobase generator (PBG) to typical $193 \mathrm{~nm}$ resist formulations was recently presented. ${ }^{1}$ Controlling the net acid concentration as a function of dose by incorporating both a photoacid generator (PAG) and a PBG in the resist formulation imparts a resist dissolution rate response modulation at twice the frequency of the aerial image. Simulation and patterning of $45 \mathrm{~nm}$ half pitch L/S patterns produced using a $90 \mathrm{~nm}$ half pitch mask were reported. ${ }^{2}$ Pitch division was achieved, but the line edge roughness of the resulting images did not meet the current standard. To reduce line edge roughness, polymer bound PBGs and polymer bound PAGs were investigated in the PDL resist formulations. The synthesis, purification, analysis, and functional performance of various polymers containing PBG or PAG monomers are described herein. Both polymer bound PBG with monomeric PAG and polymer bound PAG with monomeric PBG showed a PDL response. The performance of the polymer bound formulations is compared to the same formulations with small molecule analogs of PAG and PBG.
\end{abstract}

Keywords: pitch division lithography, line edge roughness, polymer bound PBG, polymer bound PAG

\section{INTRODUCTION}

Various processing approaches to enhancing the resolution of current $193 \mathrm{~nm}$ lithography tools have been developed. These include double patterning ${ }^{3}$, self-aligned spacer ${ }^{4}$, dual-tone development ${ }^{5}$, and use of dual-tone photoresists ${ }^{1.2}$. Most of these methods require extra processing steps and therefore have high processing costs. Pitch doubling lithography (PDL) using a dual-tone photoresist for pitch division assisted by photobase generator (PBG) was recently presented. ${ }^{1}$ By using dose-dependent base quenching to control the net acid concentration in resist films, the resist solubility response can have twice the frequency of the aerial image. In the low dose region (below $\mathrm{E}_{0}$ ) the amount of base produces is less than the acid $\left(\mathrm{k}_{\mathrm{b}}<<\mathrm{k}_{\mathrm{a}}\right)$ hence, the film behaves like a positive tone resist. In the high dose region (above $E_{n}$ ) the amount of base generated from PBG is higher than that of the acid. Therefore the net acid concentration is below the dissolution threshold, and the resist responds in negative tone. This concept is illustrated in Figure 1. This approach is compatible with the current lithography tools and process steps, and does not require extra steps, or new develop processes.

The simulation and lithography results of $45 \mathrm{~nm}$ half-pitch L/S patterns produced using a $90 \mathrm{~nm}$ half-pitch mask were reported. $^{2}$ Even though pitch division was achieved, the line edge roughness (LER) of the resulting pattern was larger than the current standard. (Figure 2) There have been many studies designed to improve LER, which can be categorized

Advances in Resist Materials and Processing Technology XXVIII, edited by Robert D. Allen, Mark H. Somervell, Proc. of SPIE Vol. 7972, 797221 · (C) 2011 SPIE · CCC code: 0277-786X/11/\$18 · doi: 10.1117/12.879771 
into two groups, one is reducing the polymer "pixel" size, and the other is binding additives to polymers to minimize diffusion. In this paper, polymer bound PBGs and polymer bound PAGs were investigated. Attaching PAGs and PBGs to polymers is expected to improve LER by decreasing the diffusion lengths of the acids and bases and by increasing the homogeneity of the photoresist formulations, and/or eliminating possible plasticization. The synthesis, purification and analysis of polymers containing PBG or PAG monomers are described. The effects of binding additives to a polymer were compared to the photoresist formulations with small molecules of PAGs and PBGs.

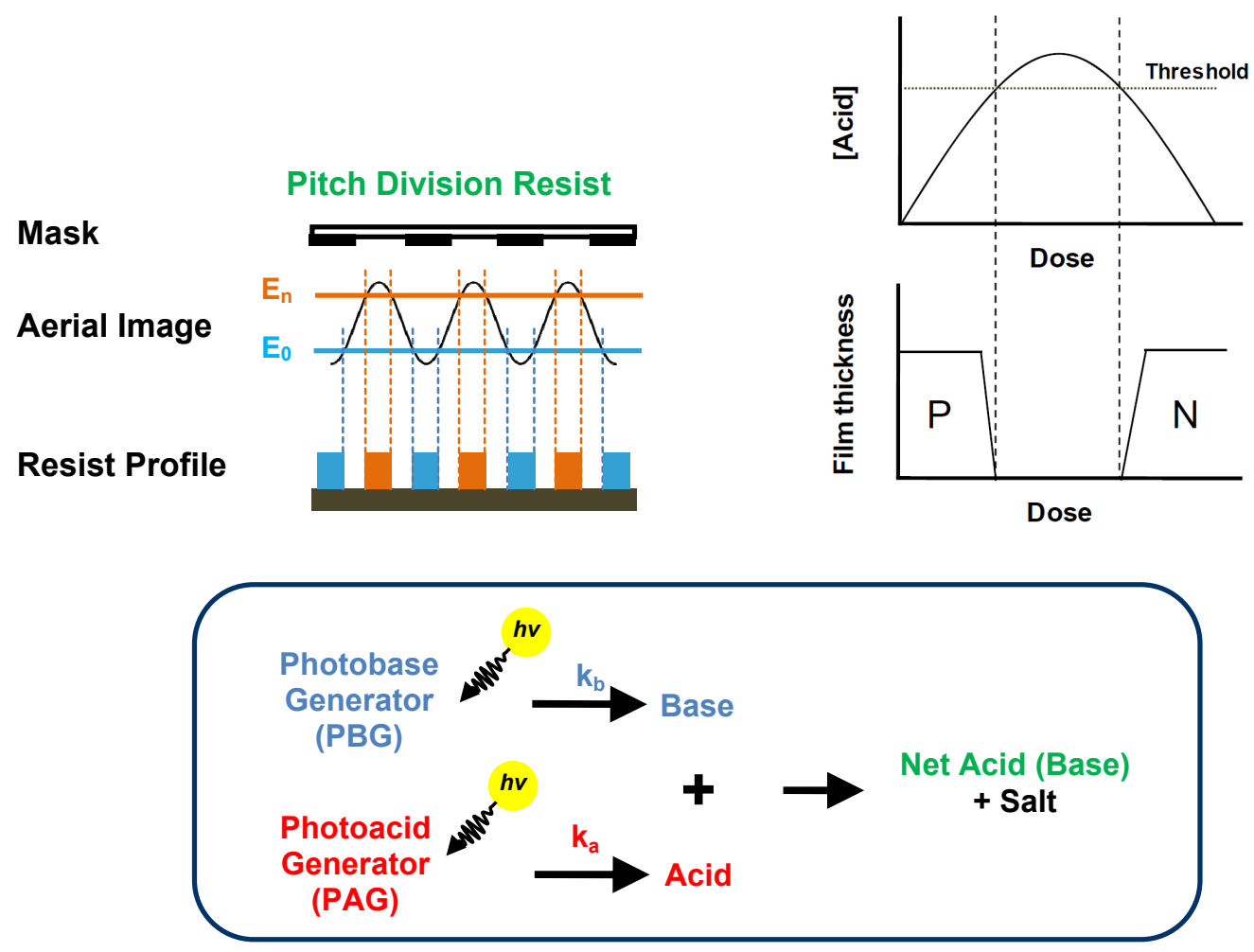

Figure 1. (Top left) An illustration of pitch division resist. In the high dose region (above $\mathrm{E}_{\mathrm{n}}$ ), the resist responds in negative tone. In the low dose region (below $\mathrm{E}_{0}$ ), the resist responds in positive tone. (Top right) To achieve a parabolic acid production curve, the key component is a PBG, which can selectively generate base to quench acid upon irradiation.
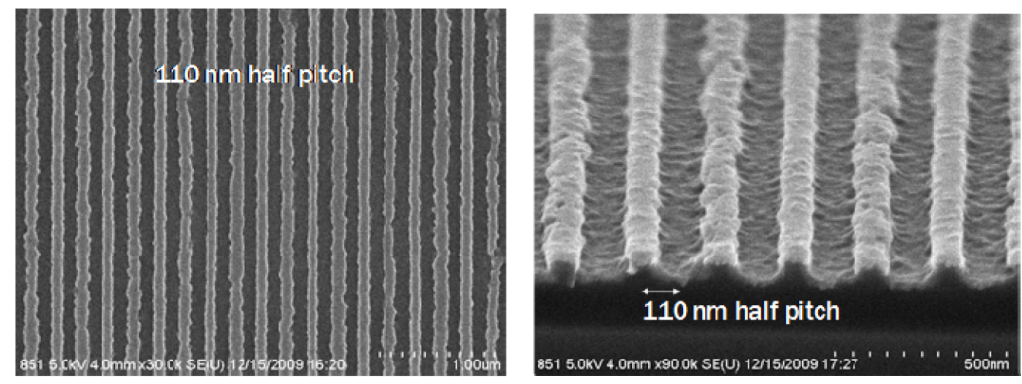

Figure 2. The SEM images of resist pattern $(110 \mathrm{~nm}$ half-pitch $)$ created on an interferometric lithography tool $(\mathrm{NA}=$ $0.22)$ using a dual-tone resist formulation. ${ }^{2}$ Note the severe LER issue. 


\section{SYNTHESIS OF POLYMER BOUND PBGs AND POLYMER BOUND PAGs}

\subsection{Synthesis of polymer bound PBGs}

An o-nitrobenzyl carbamate type PBG was chosen based on the published results. ${ }^{2}$ The mechanism of the photolysis that produces base is well characterized. ${ }^{6}$ The target PBG monomer, 2NO was synthesized as shown in Figure 3. A solution of o-nitrobenzyl alcohol $(15.3 \mathrm{~g}, 100 \mathrm{mmol})$ in dioxane $(200 \mathrm{~mL})$ was cooled to $0{ }^{\circ} \mathrm{C}$. Phosgene $(1.4 \mathrm{eq}$. as $20 \%$ in toluene) was added to the cooled solution dropwise and the resulting mixture was stirred for 3 hours at $0{ }^{\circ} \mathrm{C}$ then heat up to $50^{\circ} \mathrm{C}$ and stirred for 2 days. The remaining phosgene was then evaporated and quenched. Without further purification, the solution was cooled to $0^{\circ} \mathrm{C}, 1.8$ eq. of $\mathrm{N}$-methyl ethanolamine was added slowly, and the mixture was stirred for 2 hours then warmed to room temperature and stirred for an additional 12 hours. The solvent was then evaporated in a rotary evaporator. The resulting mixture was dissolved in diethyl ether, then washed with saturated $\mathrm{NaHCO}_{3}$ and brine, and dried over $\mathrm{MgSO}_{4}$. The solvent was evaporated in a rotary evaporator, gave $19.8 \mathrm{~g}$ of 2-nitrobenzyl 2hydroxyethyl(methyl)carbamate $\quad(78 \mathrm{mmol}, \quad$ yield: $78 \%), \quad$ as a white solid. 2-Nitrobenzyl 2hydroxyethyl(methyl)carbamate was dissolved in $250 \mathrm{ml}$ tetrahydrofuran (THF). To this solution was added 1.3 eq of triethylamine. The resulting solution was cooled to $0{ }^{\circ} \mathrm{C}$ and then 1.2 eq. of methacryloyl chloride was added dropwise. The mixture was stirred for 3 hours at $0{ }^{\circ} \mathrm{C}$, warmed to room temperature, and stirred for an additional 12 hours. The solvent was then evaporated in a rotary evaporator. The residue was dissolved in diethyl ether, washed with saturated $\mathrm{NaHCO}_{3}$ and brine, and dried over $\mathrm{MgSO}_{4}$. The solvent was evaporated in a rotary evaporator. Column chromatography was performed on the residue using EtOAc/Hex (1:3) to give 17.1 g of PBG monomer, 2-NO (53 mmol, yield: 68\%).

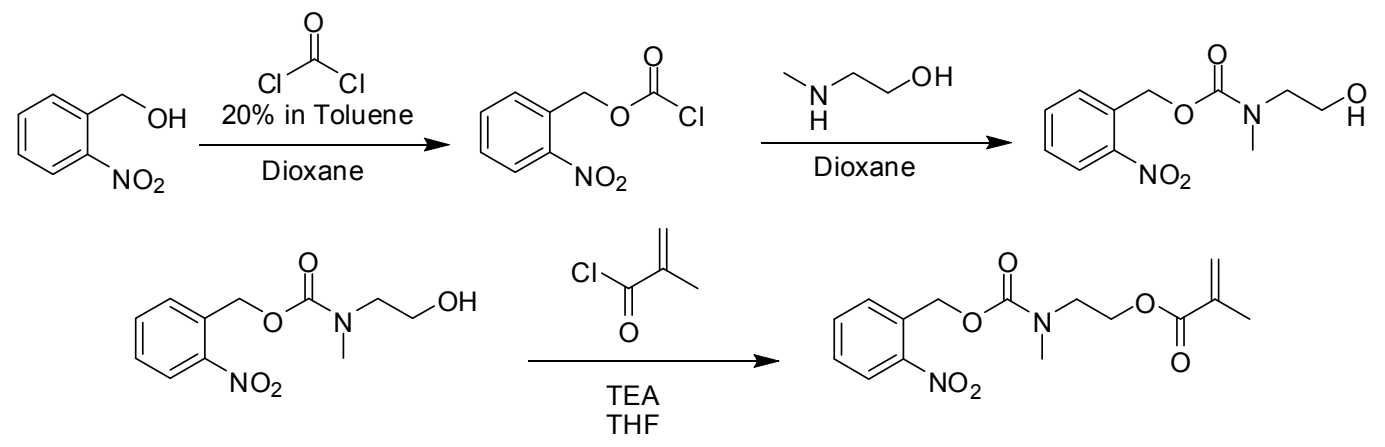

Figure 3. A synthesis scheme of PBG monomer, 2-NO.
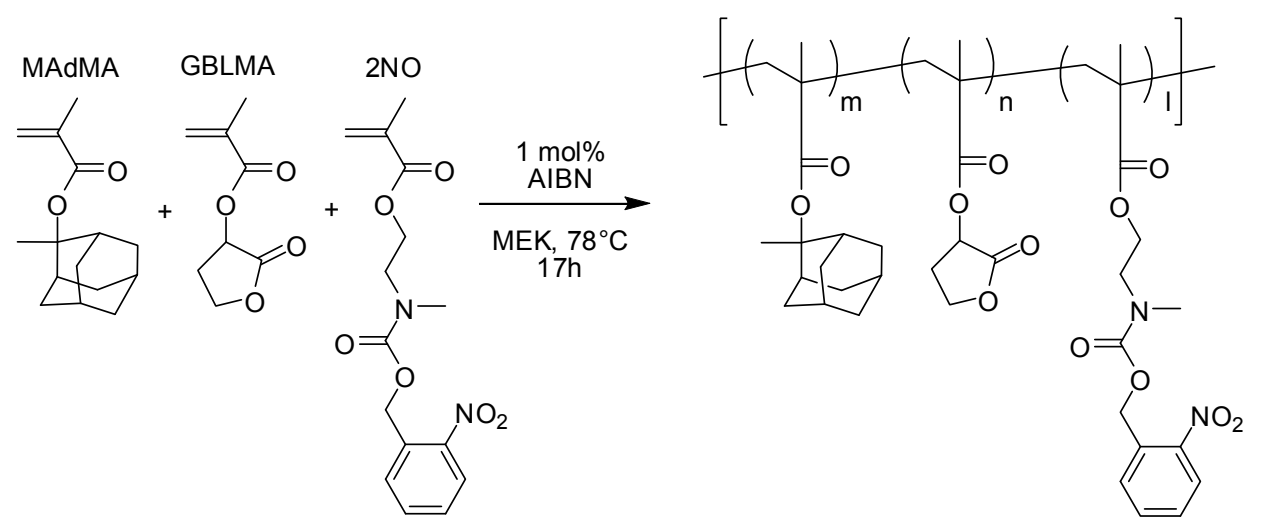

Figure 4. A polymerization scheme of PBG bound polymer. 
A polymerization reaction using the synthesized PBG monomer was performed as shown in Figure 4 . The feeding ratio was based on the previously optimized PBG loading ${ }^{2}$ (MAdMA: GBLMA: $2 \mathrm{NO}=52.2: 37.8: 10 \mathrm{in}$ mol\%.) The resulting polymer was purified by multiple precipitations. (THF: $\mathrm{MeOH}=1: 10 \mathrm{in} w \mathrm{w}$ ) The actual monomer ratios in the PBG containing polymers were determined by ${ }^{1} \mathrm{H}$ NMR, using 1 ,4-dimethoxybenzene as an internal standard. $(\mathrm{m}=47.8, \mathrm{n}=$ $42.9,1=9.3$ in mol ratio)

\subsection{Synthesis of polymer bound PAGs}

A TPS type PAG was chosen based on the published results. ${ }^{2}$ The PAG monomer, TDF was a generous gift from Central Glass, Ltd in Japan. A polymerization reaction using the PBG monomer was performed as shown in Figure 5. The feed ratio was determined based on the published results ${ }^{2}$, for the optimized PAG loading. (MAdMA: GBLMA: TDF $=55.9$ : 40.5: $3.6 \mathrm{in} \mathrm{mol \% ).} \mathrm{The} \mathrm{resulting} \mathrm{polymer} \mathrm{was} \mathrm{purified} \mathrm{by} \mathrm{multiple} \mathrm{precipitations} \mathrm{(THF:} \mathrm{MeOH}=1: 10 \mathrm{in} w \mathrm{t}$ ). The actual monomer ratios in the PAG containing polymers were determined by ${ }^{1} \mathrm{H} N \mathrm{NMR}$, using 1,4-dimethoxybenzene as an internal standard $(\mathrm{m}=56.9, \mathrm{n}=39.5, \mathrm{l}=3.6$ in mol ratio $)$.
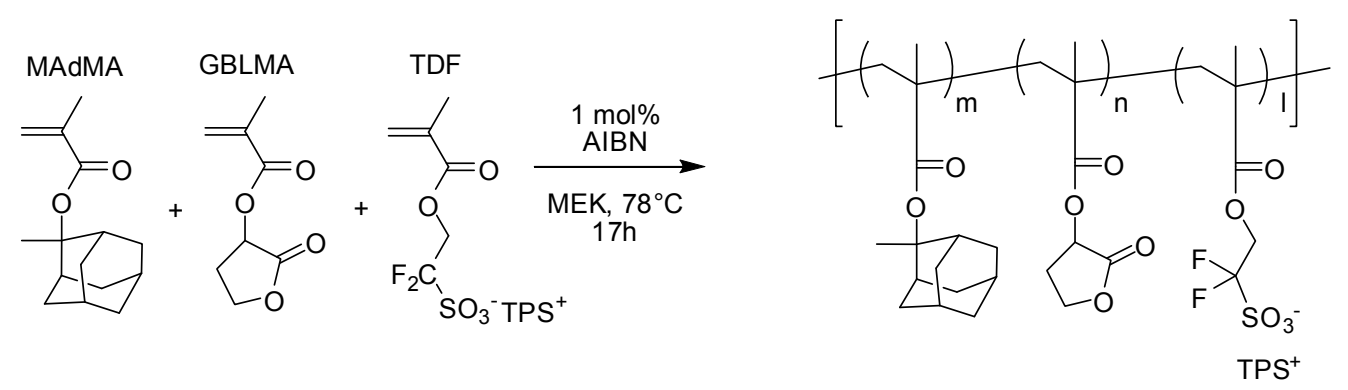

Figure 5. A polymerization scheme of PBG bound polymer.

\subsection{Synthesis of PBG monomer and PAG monomer analogs}

Analogs of the PBG monomer, 2NO, and PAG monomer, TDF, were synthesized according to the procedures, shown in Figure 6, and called 2-NO-M and TDF-M, respectively.

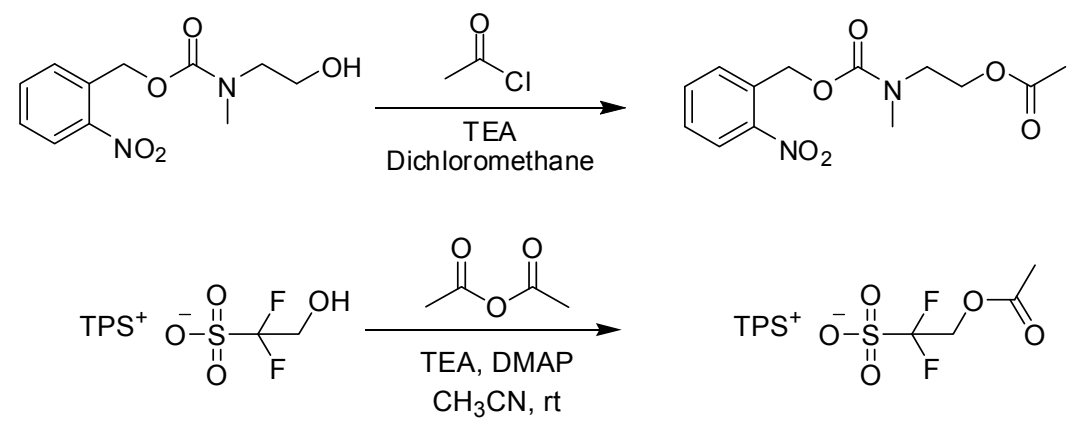

Figure 6. A synthesis scheme for analogs of the PBG monomer and the PAG monomer, 2-NO-M and TDF-M.

\subsection{Determination of residual PBG or PAG monomers in polymers}

It is critical to confirm that there is no remaining PBG or PAG monomer in polymer bound PBG and PAGs after purification. For the PAG bound polymer, the absence of unreacted PAG monomer was confirmed by ${ }^{19} \mathrm{~F}-\mathrm{NMR}$. The ${ }^{19}$ F-NMR spectrum of the PAG bound polymer did not show discernable peaks in the -114.4 ppm region, the chemical 
shift of TDF. To prove that the amount of residual PAG monomer was less than $1 \%$ of the initial feed amount, 0.057 $\mathrm{wt} \%$ of TDF monomer was added to the polymer, and the ${ }^{19} \mathrm{~F}-\mathrm{NMR}$ of the mixture was measured. The mixture has distinctive peaks in the $-114.4 \mathrm{ppm}$ region. This result showed that after purification, the PAG bound polymer contains an amount of residual PAG monomer that is less than $1 \%$ of the initial feed amount. (Figure 7)

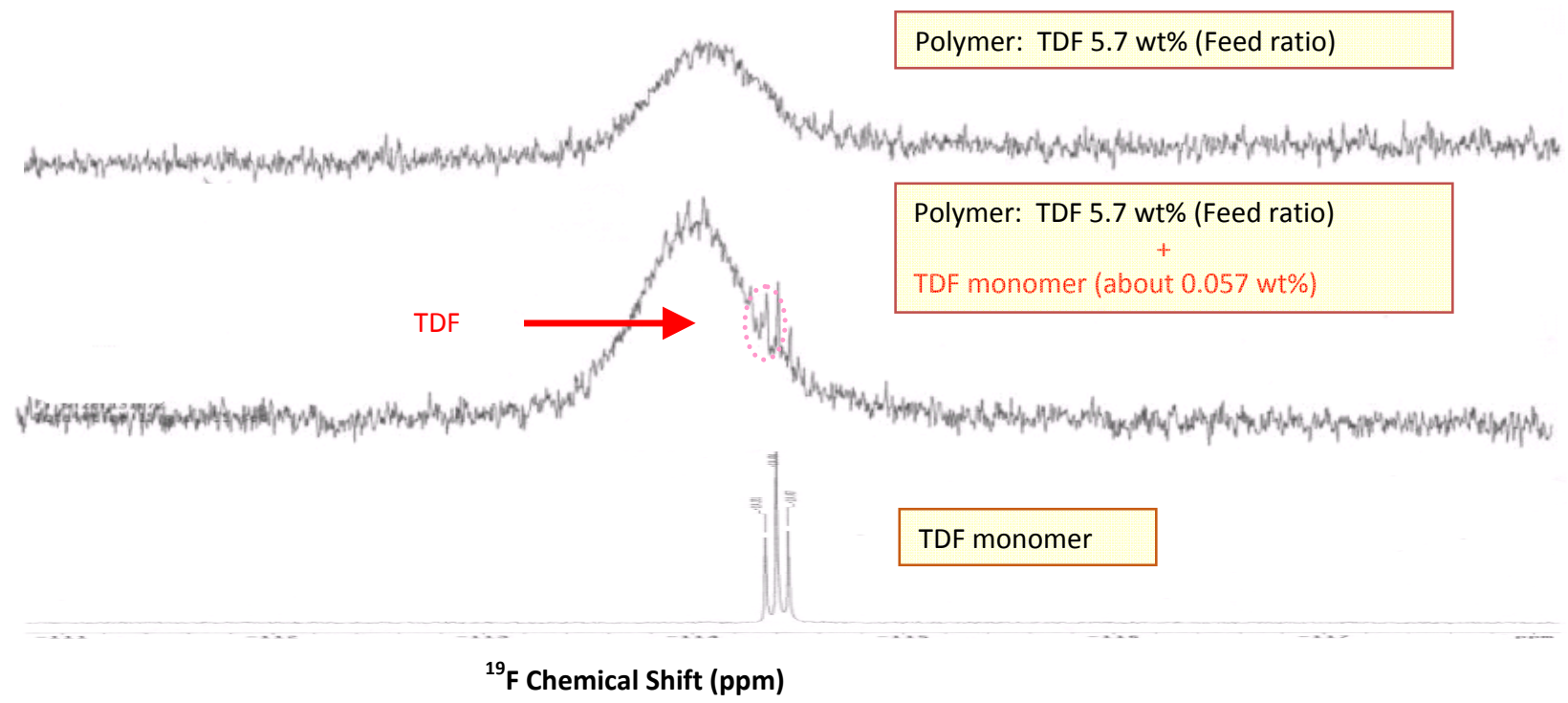

Figure 7. ${ }^{19}$ F-NMR spectra of PAG bound polymer (top), PAG monomer (TDF, bottom), and the mixture (middle).

For the PBG bound polymer, NMR was not sensitive enough to determine the amount of residual PBG monomer. . Therefore UV was used to test the purification by multiple precipitations. Twenty percent of the polymerization feed of 2-NO was added to a $15 \mathrm{wt} \%$ THF solution of copolymer, DuP (MAdMA:GBLMA=50:50). The mixture as then subjected to multiple precipitations using THF:MeOH $=1: 10(\mathrm{wt})$. After each precipitation, the UV spectrum of the filtrate was measured (Figure 8). The residual PBG monomer was about 1/120 1/140 of the initial loading after 3 precipitations. This result showed that most of residual PBG monomers were removed from the polymer during multiple precipitations, and the amount remaining would be lower than $0.2 \%$ of the initial feed. .
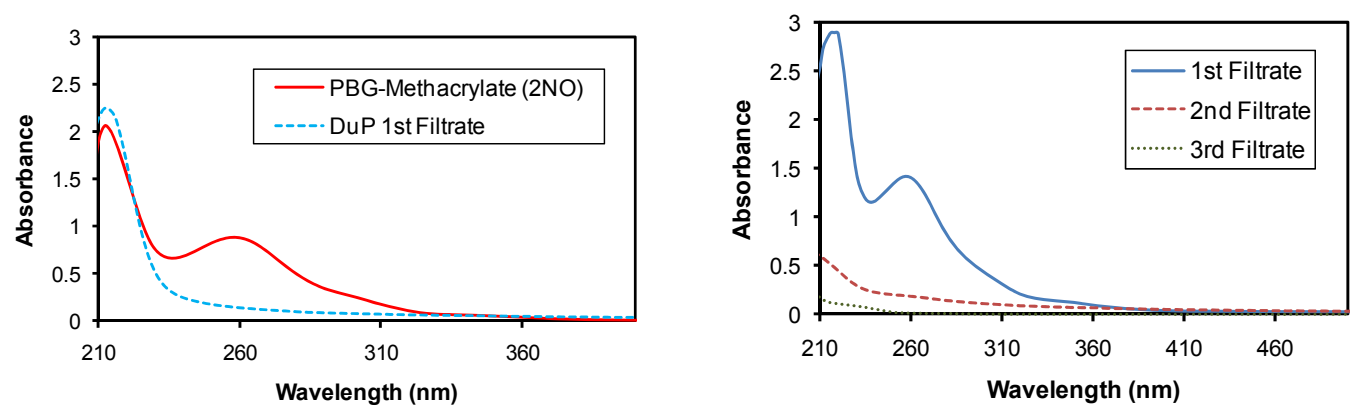

Figure 8. (Left) UV spectra of PBG monomer (2-NO) and a filtrate of copolymer (DuP) only, after the first precipitation. The DuP filtrate showed a background absorbance at $260 \mathrm{~nm}$. (Right) The UV spectra of the filtrate after each precipitation. The absorbance at $260 \mathrm{~nm}$ was 1.42 for the first, 0.19 for the second, and 0.01 for the third filtrate. 


\section{LITHOGRAPHY RESULTS}

The performance of the polymer bound formulations was compared to the same formulations made with small molecule analogs of the PAG and the PBG. The resin was first dissolved in 2-heptanone to form a $5 \mathrm{wt} \%$ solution. The amount of monomeric PBG and PAG was calculated based 3:1 molar ratio of PBG to PAG. The appropriate amounts of photoactive compound were then added to the polymer solution to formulate the final sample solutions. For monomeric PBG and PAG, the concentration of each additive was adjusted to be as close to the polymer bound cases as possible. (Table 1)

Table 1. Formulations with the polymer bound PBG, polymer bound PAG, and base polymer with monomeric PBG.

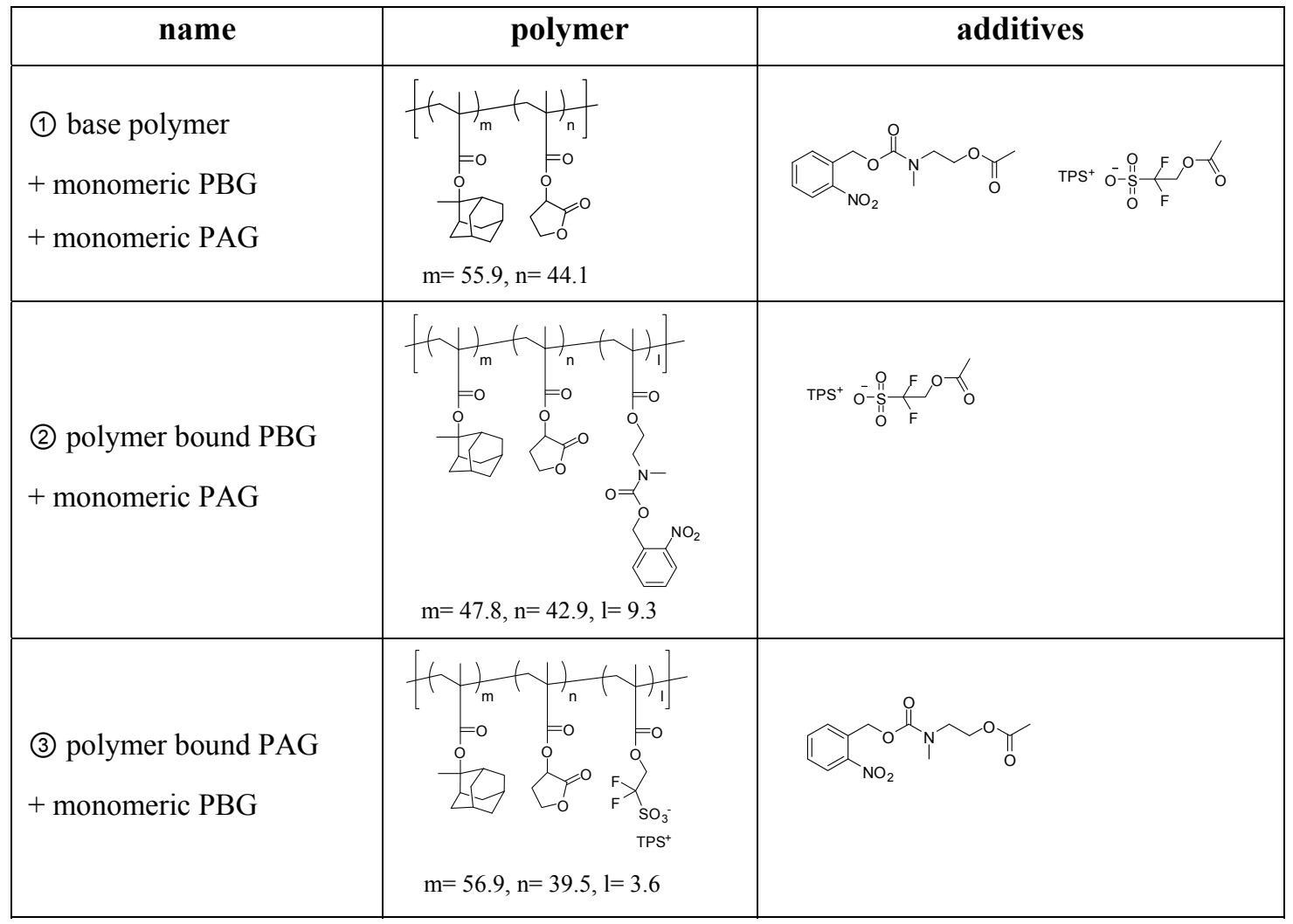

The contrast curves for the sample formulations described above at the standard condition $(120 \mathrm{~nm}$ film thickness, PAB at $110^{\circ} \mathrm{C}$ for $60 \mathrm{sec}$, exposed using $193 \mathrm{~nm}$ laser, PEB at $120^{\circ} \mathrm{C}$ for $60 \mathrm{sec}$, develop in $2.38 \%$ TMAH for $60 \mathrm{sec}$ ) are shown in Figure 9. Both polymer bound PBG with monomeric PAG and polymer bound PAG with monomeric PBG showed dual tone responses. The PBG bound polymer was expected to show lower $E_{o}$ and higher $E_{n}$ due to the confined mobility of the base. However, the opposite result was observed. This could be explained by the different dissolution behavior of the PBG containing polymer. When the PBG monomers were incorporated, the polarity and hydrophobicity of the polymer were very different from those of the base polymer. Further optimization of the comparison formulations is needed to determine the effects of bound additives.

A patterning test was performed using a $150 \mathrm{~nm} \mathrm{L/S} \mathrm{mask} \mathrm{with} \mathrm{an} \mathrm{NA} 0.85$ lens to compare bound and non-bound PBG formulations. (Figure 10) The polymer bound PBG formulation showed a better dose process window (over 16\%) than the monomeric PBG case (less than 5\%). A base quencher was added to control the sensitivity of each formulation. 

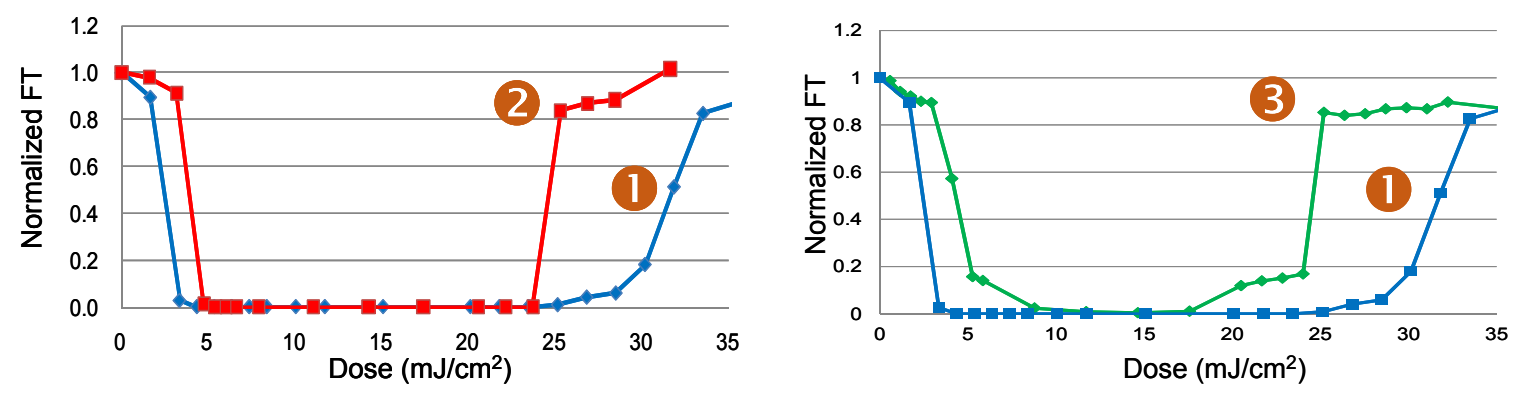

Figure 9. A contrast curve comparison of bound and non-bound PBG formulations (left) and bound and non-bound PAG formulations (right). The remaining thickness after develop was normalized.
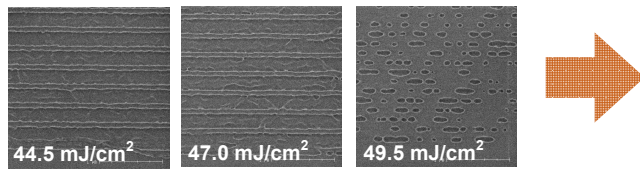
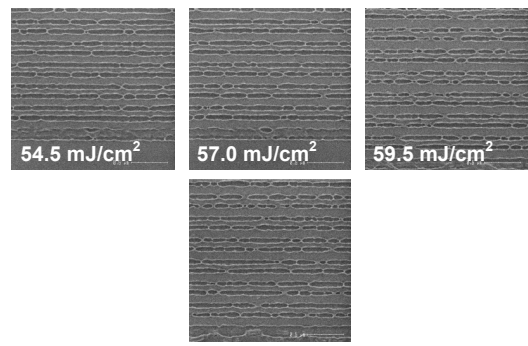

Figure 20. A process window comparison of non-bound PBG (left) and bound PBG (right) formulations. $75 \mathrm{~nm} \mathrm{L/S}$ lines were obtained using $150 \mathrm{~nm} \mathrm{L/S} \mathrm{mask.}$

\section{CONCLUSION}

A simple pitch doubling lithography technique was introduced that has the potential for extending the resolution capability of current tools without any extra processing steps. The concept is based on controlling the net acid concentration as a function of dose by incorporating both a PAG and a PBG in the resist formulation. This imparts a dissolution response modulation at twice the frequency of the aerial image. Even though pitch division was achieved, the line edge roughness (LER) of the resulting pattern was greater than the current standard. Polymer bound PBGs and polymer bound PAGs were investigated in attempt to improve the LER. Monomers of PBG or PAG, and their analogs were synthesized, and those monomers were incorporated into polymers. Polymers were analyzed to determine the concentration of residual PBG or PAG monomers and the amounts of remaining monomers was found to be negligible. Both polymer bound PBG with monomeric PAG and polymer bound PAG with monomeric PBG showed a PDL response as expected. The quality of resulting patterns and process windows were improved by using polymer bound PBGs, however, further improvement in LER is needed. Current efforts are focused on enhancing chemical contrast by developing 2-stage PBGs. 


\section{REFERENCES}

[1] X. Gu, C. Bates, Y. Cho, E. Costner, F. Marzuka, T. Nagai, T. Ogata, C. Shi, A.K. Sundaresan, N.J. Turro, R. Bristol, P. Zimmerman, and C.G. Wilson, "A New Materials-based Pitch Division Technique", J. Photopolym. Sci. and Technol. 22(6), 773-781 (2009).

[2] X. Gu, C. Bates, Y. Cho, T. Kawakami, T. Nagai, T. Ogata, A.K. Sundaresan, N.J. Turro, R. Bristol, P. Zimmerman, and C.G. Wilson, "Photobase Generator Assisted Pitch Division", Proc. of SPIE 7639, 763906 (2010).

[3] M. Hori, T. Nagai, A. Nakamura, T. Abe, G. Wakamatsu, T. Kakizawa, Y. Anno, M. Sugiura, S. Kusumoto, Y. Yamaguchi, T. Shimokawa, "Sub-40nm half-pitch double patterning with resist freezing process", Proc. SPIE 6923, 69230H (2008).

[4] W. Shiu, H. Liu, J. Wu, T. Tseng, C. Te Liao, C. Liao, J. Liu, T. Wang, "Advanced self-aligned double patterning development for sub-30-nm DRAM manufacturing", Proc. SPIE 7274, 72740E (2009).

[5] C. Fonseca, M. Somervell, S. Scheer, W. Printza, K. Nafusb, S. Hatakeyamab, Y. Kuwahara, T. Niwa, S. Bernard, R. Gronheid, "Advances and challenges in dual-tone development process optimization", Proc. SPIE 7274, 72740I-1 (2009).

[6] F.M. Houlihan, A. Shugard, R. Gooden, and E. Reichmanis, "Nitrobenzyl Ester Chemistry For Polymer Processes Involving Chemical Amplification", Macromolecules 21(7), 2001-2006 (1988). 